\title{
ANALYSIS OF FREE TORSIONAL VIBRATION IN CARBON NANOTUBES EMBEDDED IN A VISCOELASTIC MEDIUM
}

\author{
Mustafa Arda', Metin Aydogdu' \\ 1 Trakya University, 22030 Balkan Yerleşkesi, Edirne, Turkey, e-mail: mustafaarda@trakya.edu.tr
}

Received: 2015.01.26

Accepted: 2015.05.08

Published: 2015.06.01

\begin{abstract}
Carbon Nanotubes (CNTs) have a great potential in many areas like electromechanical systems, medical application, pharmaceutical industry etc. The surrounding physical environment of CNT is very important on torsional vibration behavior of CNT. Damping and elastic effect of medium to the torsional vibration of CNTs are investigated in the present study. Governing equation of motion of nanotube is obtained using Eringen's Nonlocal Elasticty Theory. The effects of some parameters like nonlocal parameter, stiffness parameter and nanotube length are studied in detail.
\end{abstract}

Keywords: torsional vibration, carbon nanotubes, nonlocal elasticity, viscoelastic medium.

\section{INTRODUCTION}

Carbon Nanotubes (CNTs) have been used in many areas because of their superior physical properties. In recent years, medical and pharmaceutical industries have great interest in CNTs. Drug transport problem to a specific tissue or cell, especially in cancer, may be solved using CNTs. Torsional dynamic behavior of CNTs in different physical environments is gaining importance in such application.

Physical environments like fluid flow or biological tissue can be modelled as a viscoelastic medium, which has damping and elastic characteristics, and the effect to the torsional vibration of CNT is a very interesting topic for researchers.

In general, CNTs can be modelled using continuum theories. Unlike from classical theory, Eringen's Nonlocal Elasticity Theory includes size effects. Eringen $[1,2]$ stated that: "The stress at a point is a functional of the strain field at every point of the continuum". Especially in nano dimensions, size dependency gains much importance because of atomic interactions.

Scientists have shown great interest in the dynamic analysis of CNTs in viscoelastic medium for years. Lots of works have been carried out.
Ghavanloo et al. investigated the vibration and instability analysis of a CNT resting on a linear viscoelastic Winkler foundation based on the classical Euler-Bernoulli beam model [3]. Shen presented buckling and postbuckling analysis for axially compressed microtubules embedded in an elastic matrix of cytoplasm. He modelled the microtubule as a nonlocal shear deformable cylindrical shell which contains small scale effects and the surrounding elastic medium as a Pasternak foundation [4]. Soltani et al. developed the transverse vibrational model of a viscous-fluidconveying SWCNT embedded in biological soft tissue. They used the nonlocal Euler-Bernoulli beam theory to investigate fluid-induced vibration of the SWCNT, while viscoelastic behavior of the surrounding tissue which is simulated by the Kelvin-Voigt model [5]. Ghavanloo et al. investigated in-plane vibration analysis of curved CNTs conveying fluid embedded in viscoelastic medium [6]. Zhen et al. studied transverse vibration of fluid-conveying DWCNTs embedded in biological soft tissue. Daneshmand studied coupled cytosol-microtubule mechanical vibrations of microtubules [7]. Kazemi-Lari et al. investigated the influence of viscoelastic foundation on the non-conservative instability of cantilever CNTs 
under the action of concentrated follower force [8]. Rafiei et al. investigated vibration characteristics of non-uniform SWCNTs conveying fluid embedded in viscoelastic medium using nonlocal Euler-Bernoulli beam theory [9]. Ghorbanpour and Arani studied nonlocal vibration of double of CNTs system conveying fluid coupled by viscoPasternak medium based on nonlocal elasticity theory where CNTs are placed in uniform temperature change and magnetic field [10]. KazemiLari et al. used the nonlocal Euler-Bernoulli beam model to predict the static and dynamic structural instability of CNTs subjected to a distributed tangential compressive load. They considered the CNT to be embedded in a Kelvin-Voigt viscoelastic medium [11]. Lei et al. investigated the dynamic behavior of nonlocal viscoelastic damped nanobeams. They employed the Kelvin-Voigt viscoelastic model, velocity dependent external damping and Timoshenko beam theory to establish the governing equations and boundary conditions for the bending vibration of nanotubes [12]. Rezaee and Maleki considered a more realistic model for the CNT conveying viscous fluid which is embedded in a viscoelastic medium [13]. Wang and Li studied the nonlinear free vibration of the nanotube with damping effects. They obtained the governing equation of the nonlinear free vibration for the nanotube based on the nonlocal elastic theory and Hamilton principle [14]. Pang et al. formulated the general governing equation of transverse wave motion in a viscoelastic SWCNT adhered by surface material on the basis of the nonlocal elasticity theory and the Kelvin model [15]. Daneshmand presented a gradient elasticity shell formulation for free vibration analysis of single-walled carbon nanotube placed on Winkler/Pasternak foundation. The proposed formulation is based on the combined strain-inertia gradient elasticity [16].

Nanocomposites are also very popular and innovative topic and some new studies are performed. Arani et al. investigated the nonlinear vibration and instability analysis of a bonded doublesmart composite microplate system (DSCMPS) conveying microflow based on nonlocal piezoelasticity theory [17]. Karličić et al. studied the free longitudinal vibration of a nonlocal viscoelastic double-nanorod system (VDNRS). They assumed that a light viscoelastic layer continuously couples two parallel nonlocal viscoelastic nanorods. Their model is aimed at representing dynamic interactions in nanocomposite materials [18].
Also boron nitride nanotubes can be used instead of carbon nanotubes. Arani et al. studied nonlinear free vibration and instability of fluidconveying double-walled boron nitride nanotubes (DWBNNTs) embedded in viscoelastic medium. They considered the effects of the transverse shear deformation and rotary inertia by utilizing the Timoshenko beam theory. Arani and Roudbari developed the nonlocal longitudinal and transverse vibrations of coupled boron nitride nanotube (BNNT) system under a moving nanoparticle using piezoelastic theory and surface stress based on Euler-Bernoulli beam [19].

Torsional dynamic analysis of CNTs can be useful for modelling in nano-products. According to author's limited literature knowledge, present topic has not been investigated yet. In next section, governing equation of motion of the CNT will be obtained using nonlocal elasticity theory.

\section{GOVERNING EQUATION OF CNT WITH VISCOELASTIC MEDIUM}

A nanotube of length $L$ and diameter $d$ is considered. If the Newton's second law is applied to nanotube for torsional vibration, the governing equation is obtained [20]:

$$
G I_{P} \frac{\partial^{2} \theta}{\partial x^{2}}=\rho I_{P} \frac{\partial^{2} \theta}{\partial t^{2}}-T_{e}(x, t)
$$

where: $G$ is the shear modulus,

$\rho$ is the density,

$I_{P}$ is the polar moment of inertia,

$\theta$ is the angular displacement of CNT.

The $I_{P}$ is defined as:

$$
I_{P}=\pi \frac{\left(R_{2}{ }^{4}-R_{1}{ }^{4}\right)}{2}
$$

where: $R_{1}$ and $R_{2}$ is the inner and outer radius of CNT.

The nonlocal constitute relation in a general form can be given as $[1,2]$ :

$$
\left(1-\mu \nabla^{2}\right) \tau_{k l}=\lambda \varepsilon_{r r} \delta_{k l}+2 G \varepsilon_{k l}
$$

where: $\tau_{k l}$ is the nonlocal stress tensor,

$\varepsilon_{k l}$ is the strain tensor,

$\lambda$ and $G$ are the Lame constants, $\mu=\left(e_{0} a\right)^{2}$ is called the nonlocal parameter, $a$ is an internal characteristic length.

$e_{0}$ is a constant - value is very important for the validity of nonlocal models.

Eringen $[1,2]$ determined this parameter with matching the dispersion curves based on the atomic models. 

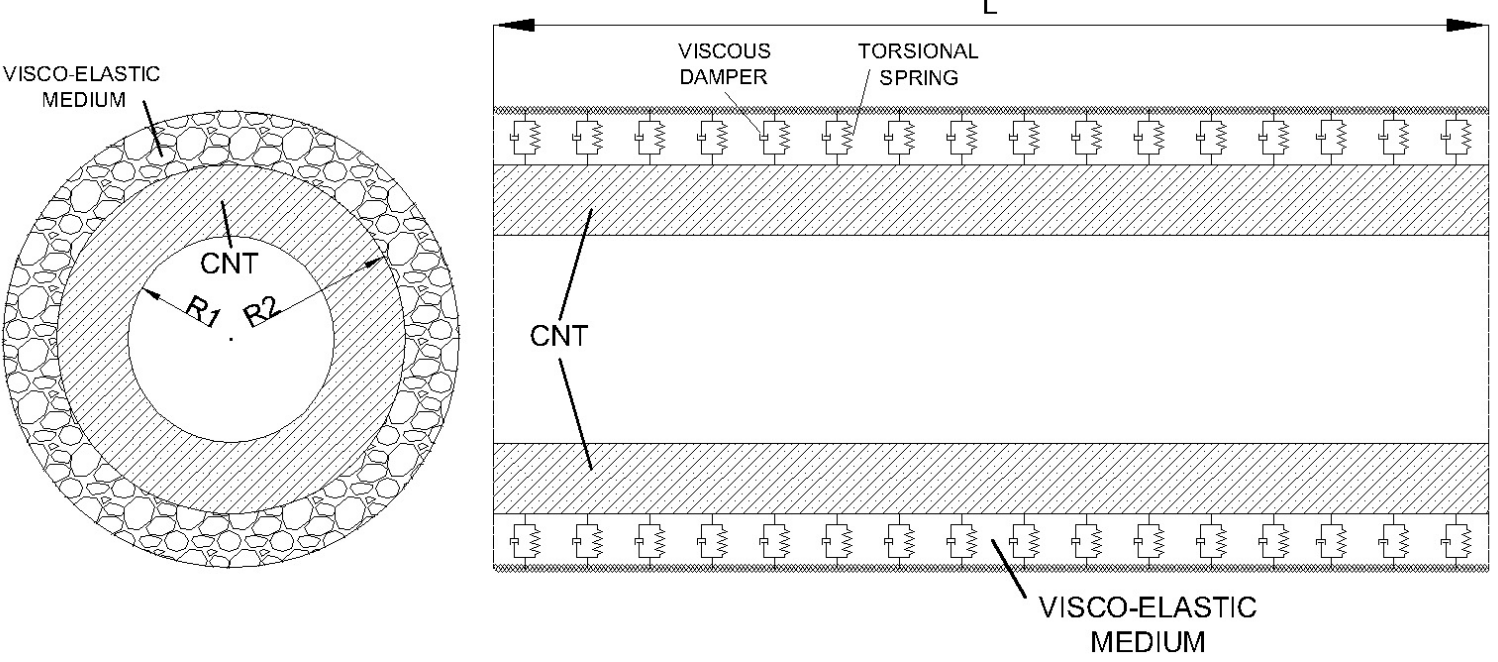

Fig. 1. CNT model with viscoelastic medium

For the torsional deformation of uniform CNT, Eq. (3) can be written in a one dimensional form:

$$
\left(1-\mu \frac{\partial^{2}}{\partial x^{2}}\right) \tau=G \gamma
$$

where: $\gamma$ is the shear strain,

$\tau$ is the shear stress of CNT.

The stress resultant due to the shear stress is expressed as:

$$
S=\int_{A} \tau d A
$$

where: $A$ is the cross-section of the CNT.

The torque relation is given as:

$$
T=\int_{A} \tau z d A
$$

By using the Eq. (4-6), we get the constitute relation as:

$$
\begin{gathered}
S-\left(e_{0} a\right)^{2} \frac{\partial^{2} S}{\partial x^{2}}=G A \gamma \\
T-\left(e_{0} a\right)^{2} \frac{\partial^{2} T}{\partial x^{2}}=G I_{P} \frac{\partial \theta}{\partial x}
\end{gathered}
$$

If Eq. (8) is inserted into Eq. (1) one obtains: $G I_{P} \frac{\partial^{2} \theta}{\partial x^{2}}=\rho I_{P} \frac{\partial^{2} \theta}{\partial t^{2}}\left(1-\mu \frac{\partial^{2}}{\partial x^{2}}\right)-T_{e}\left(1-\mu \frac{\partial^{2}}{\partial x^{2}}\right)$

If the nonlocal parameter is chosen as $\mathrm{m}=0$ in Eq. (9), the classical elasticity equation is obtained respectively. $T_{e}$ defines the external torque load and means elastic and damping effect of viscoelastic medium in this study.

$$
T=k_{t} \theta+c_{t} \frac{\partial \theta}{\partial t}
$$

where: $k_{t}$ is the stiffness,

$c_{t}$ is the damping coefficient of viscoelastic medium.
If Eq. (10) is inserted into Eq. (9), Eq. (11) is obtained:

$$
\begin{gathered}
G I_{P} \frac{\partial^{2} \theta}{\partial x^{2}}-\rho I_{P} \frac{\partial^{2} \theta}{\partial t^{2}}+\mu \rho I_{P} \frac{\partial^{4} \theta}{\partial x^{2} \partial t^{2}}+k_{t} \theta+ \\
\quad+c_{t} \frac{\partial \theta}{\partial t}-\mu k_{t} \frac{\partial^{2} \theta}{\partial x^{2}}-\mu c_{t} \frac{\partial^{3} \theta}{\partial x^{2} \partial t}=0
\end{gathered}
$$

Eq. (11) is the governing equation of motion for CNT embedded in a viscoelastic medium. With harmonic vibration assumption, angular displacement of CNT can be defined as in Eq. (12):

$$
\theta(x, t)=A(x) e^{\lambda t}
$$

where: $A(x)$ is the amplitude of torsional displacement,

$\lambda$ is the characteristic parameter for CNT.

If Eq. (12) is inserted into Eq. (11) with dimensionless parameter assumption, Eq. (13) is obtained:

$$
\begin{gathered}
\frac{\partial^{2} \theta}{\partial \bar{x}^{2}}\left[1+\frac{\mu}{L^{2}} \frac{\rho I_{P} \lambda^{2} L^{2}}{G I_{P}}-\frac{\mu}{L^{2}} \frac{c_{t} \lambda L^{2}}{G I_{P}}-\frac{\mu}{L^{2}} \frac{k_{t} L^{2}}{G I_{P}}\right]+ \\
+\theta\left[-\frac{\rho I_{P} \lambda^{2} L^{2}}{G I_{P}}+\frac{c_{t} \lambda L^{2}}{G I_{P}}+\frac{k_{t} L^{2}}{G I_{P}}\right]=0
\end{gathered}
$$

If Eq. (13) is reorganized, Eq. (14) is obtained:

where:

$$
\frac{\partial^{2} \theta}{\partial \bar{x}^{2}}+\beta^{2} \theta=0
$$

$$
\beta^{2}=\frac{-\lambda^{2} \Omega+\lambda C+K}{1-\frac{\mu}{L^{2}}\left[-\lambda^{2} \Omega^{2}+\lambda C+K\right]}
$$

where: $\Omega$ is the frequency parameter coefficient, $C$ is the damping parameter coefficient, $K$ is stiffness parameter of CNT as defined in Eq. (16):

$\Omega=\frac{\rho I_{P} L^{2}}{G I_{P}}, C=\frac{c_{t} L^{2}}{G I_{P}}, K=\frac{k_{t} L^{2}}{G I_{P}}$ 
for Clamped-Clamped (C-C) boundary condition, can be found. If Eq. (16) is rearranged, Eq. (17) can be obtained:

$$
\left[\left(1+\beta^{2} \frac{\mu}{L^{2}}\right)\left(\lambda^{2} \Omega-\lambda C-K\right)\right]+\beta^{2}=0
$$

Roots of the characteristic equation in Eq. (17), are given the characteristic value for CNT embedded in a viscoelastic medium. can be defined as non-dimensional characteristic parameter. Its imaginary part can be defined as the nondimensional damping (NDD) and real part can be defined as the non-dimensional frequency (NDF), respectively.

\section{NUMERICAL RESULTS AND DISCUSSION}

In this section, torsional vibration analysis of CNT embedded in a viscoelastic medium is carried out for different stiffness parameters, nanotube length and nonlocal parameters. Material properties accepted as shear modulus $G=$ $0.46 \mathrm{TPa}$, density $\rho=1300 \mathrm{~kg} / \mathrm{m}^{3}$, poisson ratio $v=0.19$, nanotube inner radius $R_{1}=0.68 \mathrm{~nm}$ and thickness of nanotube $h=0.066 \mathrm{~nm}$.

Validation of the present nonlocal EulerBernoulli model is investigated by authors in Ref. [20]. According to longitudinal wave propagation, similar results are found. In Figure 2, changing of torsional propagation frequency with wave number can be seen. End of the first Brillion zone, good agreement is observed with Lattice Dynamic results.

In Figures 3-5, changing of NDF with nonlocal parameter $(\mu)$ for different stiffness parameter $(K)$ and nanotube length $(L)$ is seen. NDF is decreased with increasing nonlocal parameter, stiffness parameter $(K)$ and nanotube length $(L)$.

In Figure 6, changing of NDD with nonlocal parameter $(\mu)$ for different stiffness parameters

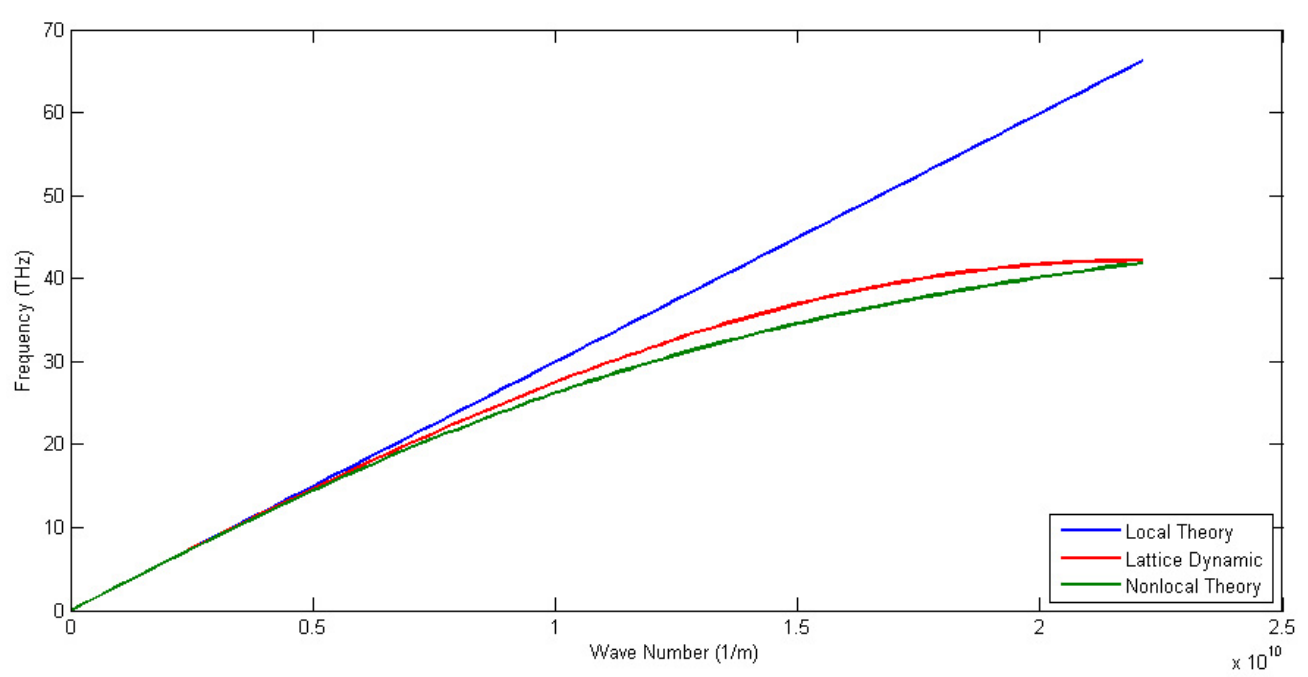

Fig. 2. Torsional wave propagation results according to different theories

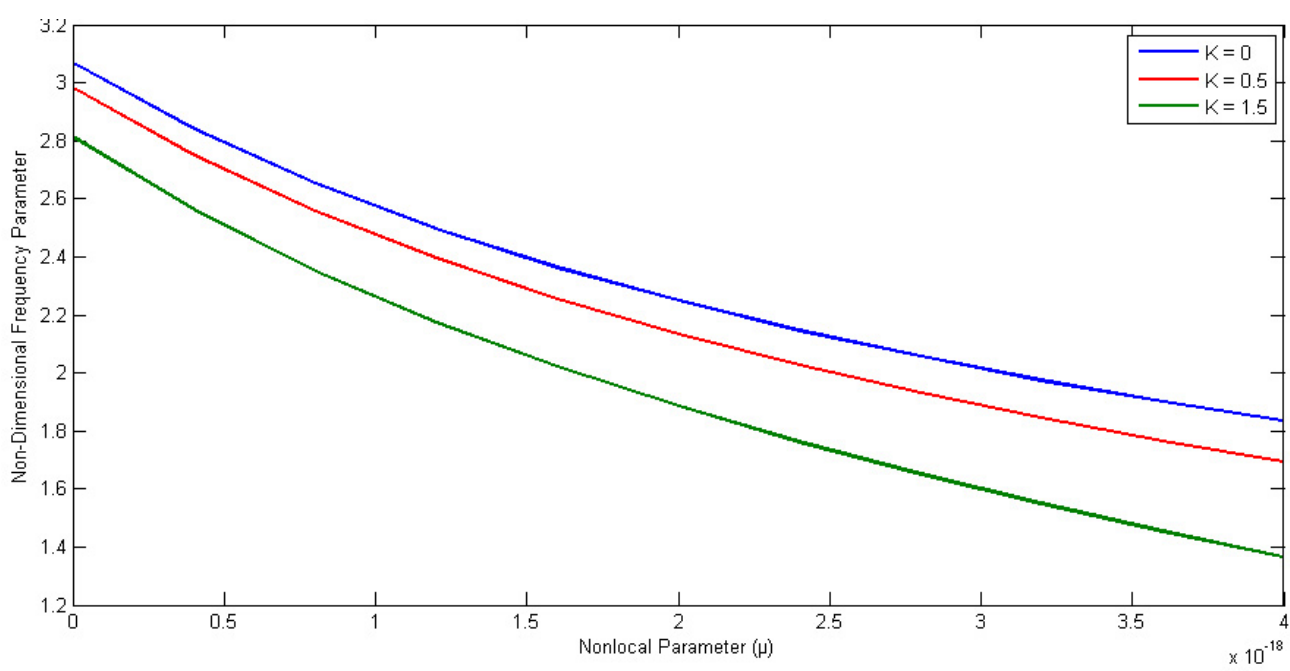

Fig. 3. Variation of NDF with nonlocal parameter $(L=5 \mathrm{~nm})$ 


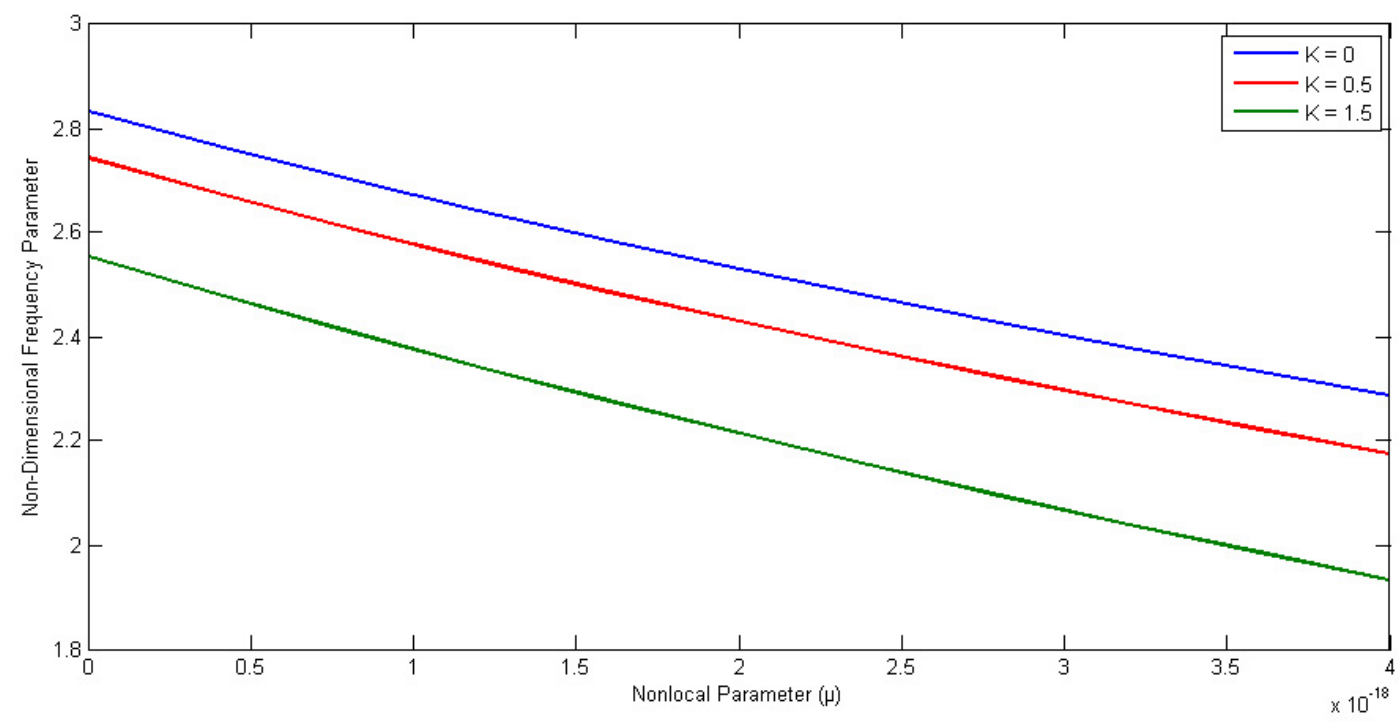

Fig. 4. Variation of NDF with nonlocal parameter $(L=10 \mathrm{~nm})$

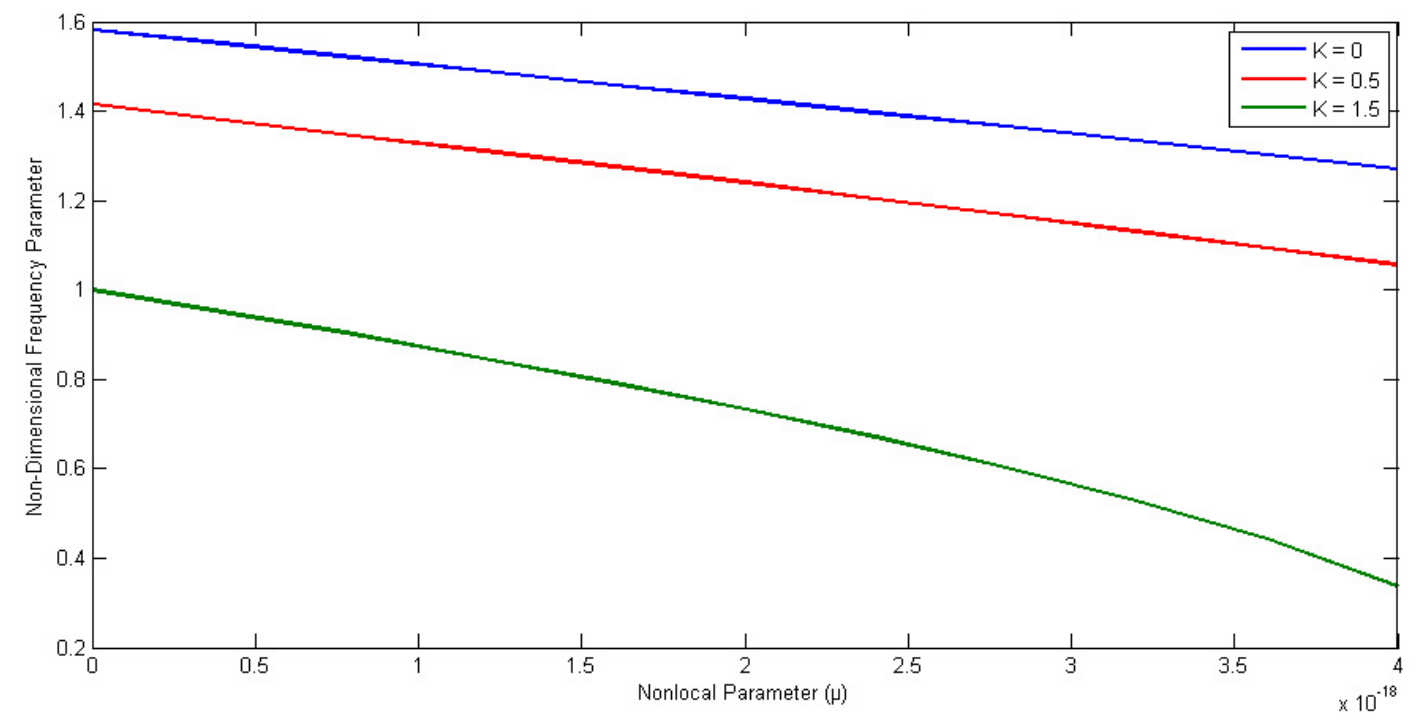

Fig. 5. Variation of NDF with nonlocal parameter $(L=20 \mathrm{~nm})$

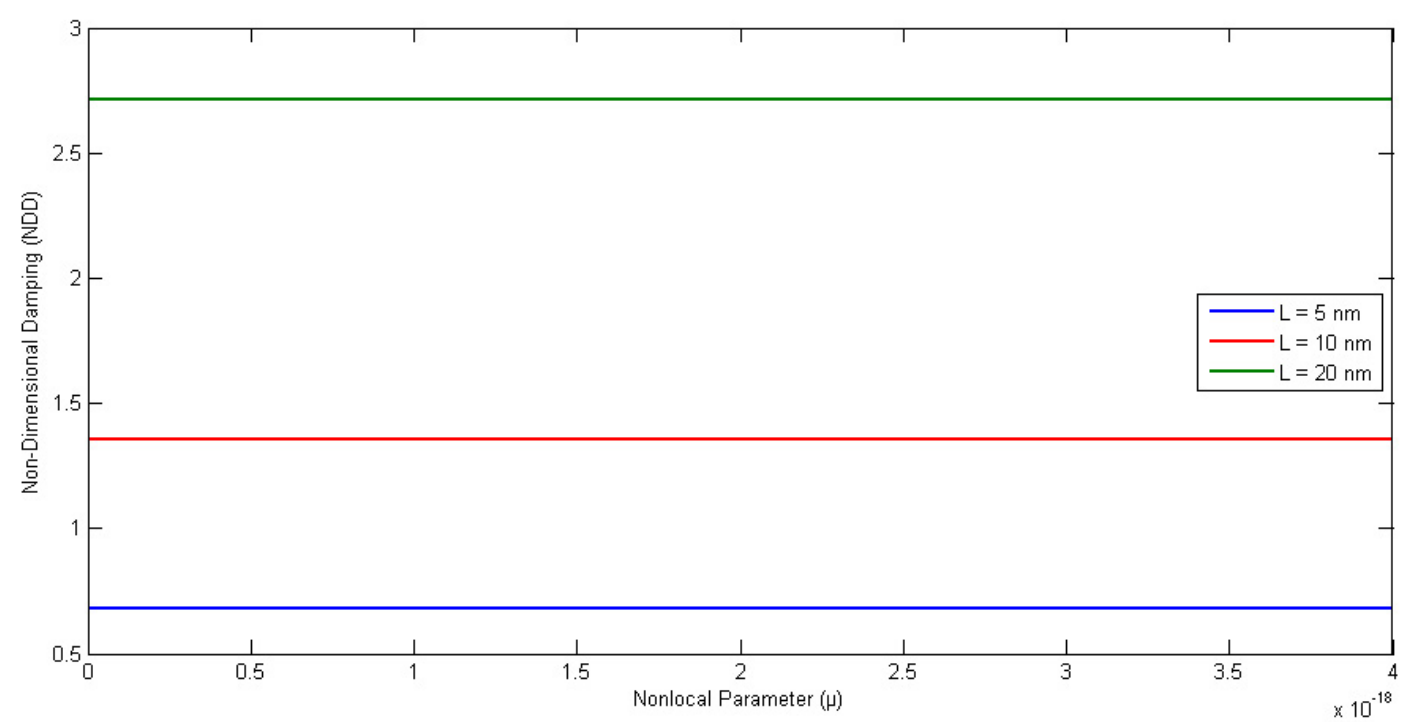

Fig. 6. Variation of NDD with nonlocal parameter 
$(K)$ and nanotube length $(L)$ is seen. NDD is not changed with increasing nonlocal parameter $(\mu)$ and stiffness parameter $(K)$; but with increased nanotube length, NDD is increased, respectively.

\section{CONCLUSION}

Free torsional vibration analysis of CNT embedded in a viscoelastic medium is investigated in detail. Governing equation of motion is obtained using Eringen's Nonlocal Elasticity Theory. Nonlocal parameter and stiffness parameter can be effected only NDF in decreasing way. Nanotube length can be effected NDF decreasingly and NDD increasingly in contrast. Present results can be useful in design of nano-electromechanical products, nano-bearings, medical applications and pharmaceutical industry, possibly.

\section{REFERENCES}

1. Eringen A.C. Nonlocal polar elastic continua. Int. J. Eng. Sci. 10, 1972, 1-16.

2. Eringen A.C. On differential equations of nonlocal elasticity and solutions of screw dislocation and surface waves. J. Appl. Phys. 54, 1983, 4703-4710.

3. Ghavanloo E., Daneshmand F., Rafiei M. Vibration and instability analysis of carbon nanotubes conveying fluid and resting on a linear viscoelastic Winkler foundation. Phys. E Low-Dimensional Syst. Nanostructures 42, 2010, 2218-2224.

4. Shen H.S. Nonlocal shear deformable shell model for postbuckling of axially compressed microtubules embedded in an elastic medium. Biomech. Model Mechanobiol. 9, 2010, 345-357.

5. Soltani P., Taherian M.M., Farshidianfar A. Vibration and instability of a viscous-fluid-conveying single-walled carbon nanotube embedded in a viscoelastic medium. J. Appl. Phys. 43, 2010, 401-425.

6. Ghavanloo E., Rafiei M., Daneshmand F. In-plane vibration analysis of curved carbon nanotubes conveying fluid embedded in viscoelastic medium. Phys. Lett. A, 375, 2011, 1994-1999.

7. Daneshmand F. Microtubule circumferential vibrations in cytosol. Proc. Inst. Mech. Eng. H, 226, 2012, 589-599.

8. Kazemi-Lari M.A., Fazelzadeh S.A., Ghavanloo E. Non-conservative instability of cantilever carbon nanotubes resting on viscoelastic foundation.
Phys. E Low-Dimensional Syst. Nanostructures 44, 2012, 1623-1630.

9. Rafiei M., Mohebpour S.R., Daneshmand F. Smallscale effect on the vibration of non-uniform carbon nanotubes conveying fluid and embedded in viscoelastic medium. Phys. E Low-Dimensional Syst. Nanostructures 44, 2012, 1372-1379.

10. Ghorbanpour Arani A. Nonlocal vibration of embedded coupled cnts conveying fluid under thermo-magnetic fields via ritz method. J. Solid Mech. 5, 2013, 206-215.

11. Kazemi-Lari M.A., Ghavanloo E., Ahmad Fazelzadeh S. Structural instability of carbon nanotubes embedded in viscoelastic medium and subjected to distributed tangential load. J Mech Sci Technol 27, 2013, 2085-2091.

12. Lei Y, Adhikari S, Friswell M.I. Vibration of nonlocal Kelvin-Voigt viscoelastic damped Timoshenko beams. Int. J. Eng. Sci. 66-67, 2013, 1-13.

13. Rezaee M., Maleki V.A. An analytical solution for vibration analysis of carbon nanotube conveying viscose fluid embedded in visco-elastic medium. Proc. Inst. Mech. Eng. Part C, J. Mech. Eng. Sci. 229, 2014, 644-650.

14. Wang Y-Z., Li F-M. 2014, Nonlinear free vibration of nanotube with small scale effects embedded in viscous matrix. Mech Res Commun 60, 45-51.

15. Pang M, Zhang Y.Q, Chen W.Q. Transverse wave propagation in viscoelastic single-walled carbon nanotubes with small scale and surface effects. J. Appl. Phys. 117, 2015, 305-324.

16. Daneshmand F. 2014, Combined strain-inertia gradient elasticity in free vibration shell analysis of single walled carbon nanotubes using shell theory. Appl Math Comput 243, 856-869.

17. Ghorbanpour Arani A., Vossough H., Kolahchi R. Nonlinear vibration and instability of a viscoPasternak coupled double-DWBNNTs-reinforced microplate system conveying microflow. Proc. Inst. Mech. Eng. Part C, J. Mech. Eng. Sci. 0, 2015, 1-17.

18. Karličić D., Cajić M., Murmu T., Adhikari S. Nonlocal longitudinal vibration of viscoelastic coupled double-nanorod systems. Eur J Mech - A/ Solids 49, 2015, 183-196.

19. Ghorbanpour Arani A., Roudbari M.A. Nonlocal piezoelastic surface effect on the vibration of visco-Pasternak coupled boron nitride nanotube system under a moving nanoparticle. Thin Solid Films 542, 2013, 232-241.

20. Arda M., Aydogdu M. Torsional statics and dynamics of nanotubes embedded in an elastic medium. Compos Struct 114, 2014, 80-91. 